\title{
Author Correction: Challenges and gaps for energy planning models in the developing-world context
}

Kumar Biswajit Debnath (D) and Monjur Mourshed (D)

Correction to: Nature Energy https://doi.org/10.1038/s41560-018-0095-2, published online 6 March 2018.

In the version of this Review originally published, in Table 3 seven entries in the columns 'Developer' and 'Country of origin' were incorrect and should have read as shown below; these errors have now been corrected. All other entries in the Table are unaffected.

\section{Table 3 | Original and corrected sections}

\begin{tabular}{|c|c|c|}
\hline Model & Developer & Country of origin \\
\hline \multicolumn{3}{|l|}{ Original } \\
\hline MARKAL-MACRO & International Energy Agency (IEA) and ETSAP & France \\
\hline MICRO-MELODIE & US Department of Energy & USA \\
\hline TIMES-MACRO & Brookhaven National Laboratory & USA \\
\hline ENPEP & Commissariat à l'énergie atomique et aux énergies alternatives (CEA) & France \\
\hline LEAP & Energy Technology Systems Analysis Program (ETSAP) and International Energy Agency (IEA) & France \\
\hline Mesap PlaNet & International Atomic Energy Agency (IAEA) & Austria \\
\hline EFOM-ENV & Stockholm Environmental Institute, Boston & USA \\
\hline \multicolumn{3}{|l|}{ Corrected } \\
\hline MARKAL-MACRO & Brookhaven National Laboratory & USA \\
\hline MICRO-MELODIE & Commissariat à l'énergie atomique et aux énergies alternatives (CEA) & France \\
\hline TIMES-MACRO & Energy Technology Systems Analysis Program (ETSAP), International Energy Agency (IEA) & France \\
\hline ENPEP & International Atomic Energy Agency (IAEA) & Austria \\
\hline LEAP & Stockholm Environmental Institute & USA \\
\hline Mesap PlaNet & Institutes für Energiewirtschaft und Rationelle Energieanwendung (IER), University of Stuttgart & Germany \\
\hline EFOM-ENV & European Commission DDG-XII F/1 & Belgium \\
\hline
\end{tabular}

